\title{
Impact of HFOSS Education on Instructors
}

\author{
Lori Postner, Darci Burdge \\ Nassau Community College \\ Garden City, NY, USA \\ lori.postner@ncc.edu \\ darci.burdge@ncc.edu
}

\author{
Heidi J. C. Ellis, Stoney Jackson \\ Western New England University \\ Springfield, MA, USA \\ ellis@wne.edu \\ stoney.jackson@wne.edu
}

\author{
Gregory W. Hislop \\ Drexel University \\ Philadelphia, PA, USA \\ hislop@drexel.edu
}

\begin{abstract}
Student involvement in Humanitarian Free and Open Source Software (HFOSS) increases student self-reported learning while providing the motivation of "doing good" [11]. Supporting this type of student participation also impacts the instructors. This effect includes modifying one's teaching approach, providing a different perspective on the subject matter, and changing how instructors perceive their roles. This paper explores the impact on instructors that result from supporting student participation in HFOSS, including consideration of pedagogical practices, self-perceptions of teaching, and exposure to open source culture and technologies. The paper also examines resources that instructors consider important for HFOSS education. The analysis presented here is based upon a qualitative analysis of semi-structured interviews with 24 faculty members. Results indicate that exploring HFOSS education changes instructors' pedagogical approaches, broadens instructors' perspectives on the connections between academic content and professional practice, and has impact on instructors' attitudes and motivations when teaching.
\end{abstract}

\section{CCS CONCEPTS}

- Social and professional topics $\rightarrow$ Computer science education.

\section{KEYWORDS}

HFOSS, Humanitarian Computing, Computing Education

\section{ACM Reference Format:}

Lori Postner, Darci Burdge, Heidi J. C. Ellis, Stoney Jackson, and Gregory W. Hislop. 2019. Impact of HFOSS Education on Instructors. In Proceedings of Innovation and Technology in Computer Science Education (ITiCSE '19). ACM, New York, NY, USA, 7 pages. https://doi.org/10.1145/3304221.3319765

\section{INTRODUCTION}

Participation in Humanitarian Free and Open Source Software (HFOSS) projects has a positive impact on student attitude towards computing $[7,11]$, perceived learning of software engineering knowledge [5], technical knowledge and professional skills [7], and understanding how to plan, develop and maintain a software project

Permission to make digital or hard copies of all or part of this work for personal or classroom use is granted without fee provided that copies are not made or distributed for profit or commercial advantage and that copies bear this notice and the full citation on the first page. Copyrights for components of this work owned by others than the author(s) must be honored. Abstracting with credit is permitted. To copy otherwise, or republish, to post on servers or to redistribute to lists, requires prior specific permission and/or a fee. Request permissions from permissions@acm.org.

ITiCSE '19, fuly 15-17, 2019, Aberdeen, Scotland UK

(c) 2019 Copyright held by the owner/author(s). Publication rights licensed to ACM ACM ISBN 978-1-4503-6301-3/19/07 . \$ \$15.00

https://doi.org/10.1145/3304221.3319765
[6]. In addition, female students report a greater understanding of project management aspects of HFOSS than do male students [6].

Given the benefits students reap from involvement in an HFOSS project, adoption of student participation in HFOSS is an attractive way to provide real-world experience to computing students. However, this participation is accompanied by a learning curve for both students and instructors [18, 22]. In addition, finding an appropriate project for a particular classroom context is challenging since the instructor must consider the language and technologies used by the project, the size of the project, and the culture of the community as well as any domain knowledge potentially needed in order to successfully contribute to it. After identifying an appropriate project, locating a mentor or contact person within the project smooths the start-up, but the right contact person may not be easy to find. Downloading and installing the project as well as learning the project work flow and processes may involve troubleshooting and investing time to learn new tools and technologies.

Although all of this may sound daunting and deter instructors from trying to incorporate HFOSS, the effort has benefits for the instructor too. Going through this process provides instructors, some of whom lack industry experience, exposure to professional practice in the open source world as well as the work environments their students will encounter upon graduation. This is a valuable experience for many instructors and helps them to keep current in the area of software development as well as to model for their students the importance of being a life long learner.

In order for student participation in HFOSS to be widely adopted, the impact on instructors of such participation must be examined in terms of both costs and benefits. An understanding of instructor experiences, specifically what it takes to successfully support student learning within HFOSS projects, will provide a solid basis for creating the right community infrastructure and instructor professional development to support expansion of student learning within HFOSS communities.

\section{RELATED WORK}

Free and Open Source Software (FOSS) projects are becoming more widely used to provide students with hands-on, real-world experience with software projects [3]. Frequently, student involvement in a FOSS project happens in Software Engineering courses [13, 19], and may also occur in lower level courses such as Data Structures [27], throughout the curriculum, [1, 2], and outside of the classroom via clubs or other extra-curricular activities [4].

HFOSS education can be viewed as one thread within the extensive body of computing education research that constitutes a shift from traditional lecture methods to forms of active learning including pair programming [26], flipped classrooms [10], blended learning [12], peer-instruction [23], problem-based learning [15] 
and others. Many of these efforts share considerations related to the goals of HFOSS education. In this broad push to improve computing education, there is general recognition that the role of the instructor changes, but there has been relatively little investigation into how this shift affects instructors' perspectives on teaching. In addition, given the rapid rate of change in technology, computing instructors have relatively high burdens to keep current in their field, which also impacts their role as instructors.

Several studies are related to the current work in that they have addressed aspects of instructor perspectives on the instructor's role, and on issues of keeping up with technology or reflecting professional practice in teaching. A few studies have provided a comparison of student and instructor beliefs and attitudes about Computer Science. Perrenet [21] and Lewis [14] studied the enculturation of undergraduate CS students by comparing how their attitudes and beliefs transform as they progress through their degree program. Both works surveyed instructors about their belief about the CS discipline, although they did not survey instructors about what and how the instructors learned as computing advances.

Porter [24] reports on an instructor boot camp that has the goals of supporting classroom practices that have been shown by evidence to be successful and to change instructor perceptions of teaching so that they view it as a scholarly effort. Porter mentions that changing teaching practices may be difficult. Simon [28] reports on instructor development workshops to increase active learning in the classroom. They report a $44 \%$ adoption rate for active learning techniques, and the influences that positively impacted instructor adoption included sufficient technology support and a community of instructors working together.

$\mathrm{Ni}[20]$ used semi-structured interviews to determine barriers and helpers for instructors to adopt curriculum changes. Eight instructors from different academic institutions were interviewed and factors that influenced curriculum change included a sense that the traditional computer science curriculum was not effective, perceived benefits for students resulting from the curriculum change, and evidence that demonstrated that the adopted curriculum was more effective than the traditional one.

Zheng [30] reports on a grounded theory investigation into the perspectives of $14 \mathrm{MOOC}$ instructors. Instructors were interviewed about their experiences in teaching MOOCs, particularly the motivations and challenges that occur when teaching MOOCs. Results identified four motivating factors that inspired instructors teaching MOOCs including professional growth, research opportunities, world-wide impact on students, and enhanced name recognition.

There has been little work done in the area of professors' perspectives on teaching open source. One effort [22] investigated the benefits, challenges, and opportunities of supporting student involvement in a FOSS project. Interviews were performed with seven Software Engineering professors from a variety of counties who included FOSS in their courses. The focus of these interviews was on how the FOSS projects were used within the course and did not cover instructor opinions on their own learning or impact on their teaching. Results indicated that instructors utilized FOSS projects in a variety of manners with a range of student learning activities. Benefits to students included improved student learning, both technical and professional, and barriers included the learning curve faced by both student and instructor. These results corroborate earlier research into student experiences with HFOSS [5-7].

\section{METHODS}

This paper focuses on the impact on instructors of supporting student participation in an HFOSS project. In particular, the following research questions motivate the analysis reported here:

- Q1: How did HFOSS education change the instructors' approaches and perspectives toward computing instruction?

- Q2: What resources did instructors believe were most important to their success in HFOSS education?

\subsection{Study Design}

The effort to expand student participation in HFOSS projects has included instructor professional development in the form of NSFfunded instructor workshops for instructors interested in incorporating HFOSS education into their courses. These Professors' Open Source Software Experience (POSSE) workshops have been held once or twice a year since 2013. Over 140 instructors from over 110 different institutions have completed the workshop. This community served as the population for this study.

The study involved an online survey followed by a semi-structured interview. The survey was intended to:

- Gather demographic information about the participants

- Construct a list of courses in which instructors successfully incorporated HFOSS

- Determine whether instructor background influenced success in HFOSS education

- Explore the hurdles instructors encountered as they incorporated HFOSS education into their courses

An analysis of survey results is reported in [25].

\subsection{Data Collection}

The goal of the semi-structured interview portion of the study was to obtain observations about the impact of supporting student involvement in HFOSS on the individual instructor. The participants were selected from instructors who had participated in a POSSE workshop and had at least one academic year after the workshop date to incorporate HFOSS into their classroom. At the start of the study, there were 77 eligible POSSE alumni who had attended a POSSE workshop at least one year in the past. This group was narrowed to include only one participant per institution, and to focus on instructors who had little or no experience with HFOSS education prior to attending the POSSE workshop, but had actively participated in the online and in-person parts of POSSE. This resulted in 41 potential participants who were contacted via email and invited to participate in the study by completing the initial survey [25]. This group included both instructors who had been successful in supporting student involvement in HFOSS and instructors who had not been successful yet. Twenty-six instructors completed the survey, a $66 \%$ response rate. Of these 26,24 agreed to be interviewed and participated in a semi-structured interview. The interviewees consisted of 11 women and 13 men, a more gender 
balanced population than the general population of computing instructors [31]. This gender ratio is comparable to the larger POSSE alumni community.

The instructors in the study came from institutions located in 16 different U.S. states. Two institutions were 2-year and the remaining 22 were 4 -year institutions. Eleven were private not-for-profit and 13 were public. There were five small institutions, eleven medium sized institutions, seven large institutions and one very large institution according to the Carnegie Classifications of Institutions of Higher Education [8]. Also according to the Carnegie Classifications, the selectivity of the institutions varied with 11 institutions being "more selective", eight being "selective", and two being "inclusive". The two 2-year schools and one of the 4-year schools were not rated based on selectivity.

As for the instructors, one was black, 21 were white and two declined to answer. Five were Assistant Professors, 11 were Associate Professors, four were full Professors and two were Professors and Chairs. In addition, there was an Associate Teaching Professor and a Clinical Assistant Professor. Table 1 shows the number of years of experience as a computing educator for the study sample. It is interesting to note that $83 \%$ of instructors had six or more years of computing education experience and over half had more than 10 years of experience. Table 2 shows the number of years of both industry and open source experience of the participating instructors.

\begin{tabular}{|c|c|}
\hline Years & \# Faculty \\
\hline $2-5$ & 4 \\
\hline $6-10$ & 5 \\
\hline$>10$ & 15 \\
\hline
\end{tabular}

Table 1: Years Experience as a Computing Educator

\begin{tabular}{|c|c|c|}
\hline Years & $\# \mathbf{w} /$ Industry & $\# \mathbf{w}$ / Open Source \\
\hline 0 & 7 & 5 \\
\hline $1-4$ & 9 & 12 \\
\hline $5-10$ & 4 & 4 \\
\hline$>10$ & 4 & 3 \\
\hline
\end{tabular}

Table 2: Industry and Open Source Experience

Faculty incorporated HFOSS into a wide range of courses at the 100, 200, 300 and 400 levels including: Object Oriented Program Design, Data Structures, Algorithms, Software Engineering, Computer Security, Senior Seminar and Mobile Development. There is some evidence that instructors teaching project courses such as Software Engineering or a capstone course find it easier to incorporate HFOSS into the classroom. The semi-structured interview focused on the instructor's experiences in the classes where they supported student involvement in HFOSS. There were four questions for instructors who had not involved students in HFOSS including "Tell us a bit more about why you haven't involved students in HFOSS." and "What could the research project team do that might help you involve students in HFOSS?" The interview for instructors who had involved students in HFOSS consisted of nine open-ended questions and included questions such as "How did your use of HFOSS work in your class?" and "What have you learned from interacting with HFOSS communities?" Interviews were conducted either face-toface or over the phone. Interviews took between 30-60 minutes. For the face-to-face interviews, two researchers were present with one researcher asking the questions and the second taking notes.

\subsection{Data Analysis}

The data was analyzed using a grounded theory process of inductive coding [16, 29] using the CATMA textual analysis tool [17]. The raw data were the notes taken during the instructor interview. An initial set of categories and codes were derived from the interview questions. Two researchers then independently went through all of the interviews, coding the data and adding codes as needed. Once all of the data was coded, axial coding was used to relate codes and categories. The codes were reviewed, common codes identified, and a hierarchy of categories was created within code sets. One researcher created the initial category and code organization and a second researcher reviewed and suggested revisions. The interview data for 24 instructors provided a large corpus for a qualitative study, where the focus is on developing understanding and insight into the questions rather than the typical goals of statistical significance sought in quantitative studies.

\section{RESULTS}

The qualitative approach outlined above identified a series of themes in the data that help provide answers to the two research questions. These themes are discussed in the sub-sections below for each research question.

\subsection{Impact on Instructors' Approach and Perspective}

This section presents emergent themes that help to answer research question 1: How did HFOSS education change the instructors' approaches and perspectives toward computing instruction? The data for these themes comes from the interviews of the 19 instructors (seven women, 12 men) who incorporated HFOSS into their courses. The remaining five participants (four women, one man) had not been successful with HFOSS education at the time of the interview. When asked why they were not able to incorporate HFOSS into courses yet, these five cited not teaching an appropriate course and not having enough time to prepare HFOSS materials as the main reasons. Faculty described involvement in a number of HFOSS projects including: Ushahidi, Mifos, OpenMRS, GNOME Mousetrap, Mozilla, and SugarLabs.

4.1.1 Instructors as Participants in HFOSS Communities. HFOSS education often requires a level of participation by the instructor that is distinctive. Five of the interviewees $(26 \%)$ mentioned their own connection to the HFOSS community in their interviews as having impacted their approach to teaching. Table 3 shows an example code and coded data for this theme. Additional codes included Impact of FOSS, Community culture/politics rate of change, Participate in community, and Persistence (with regard to interacting with the community).

Five interviewees made 12 observations about some aspect of HFOSS community participation in their interviews and one specifically stated that $\mathrm{s} /$ he did not interact with the HFOSS community. One of the most common discussion points related to the impact of the 


\begin{tabular}{|l|l|}
\hline Code & Raw Data \\
\hline $\begin{array}{l}\text { Importance of } \\
\text { community }\end{array}$ & $\begin{array}{l}\text { "You have to interact with the community } \\
\text { as a professor." }\end{array}$ \\
\hline
\end{tabular}

Table 3: Example Coding for Instructors as Participants

FOSS community on learning. Finding an appropriate FOSS project community was identified as important as evidenced by statements such as "It is important to find the right community, some are more welcoming, some less". Finding the right HFOSS community can be a challenge for a number of reasons. As another participant pointed out, the project politics may make it difficult: "their [the project's] internal politics and dynamics changes a lot over time ... they were in a midst of a leadership meltdown of sorts". Another participant stated how important it is for the professor to interact so that $\mathrm{s} / \mathrm{he}$ can understand the communications that occur and focus on working with community members who are positive "Having some people that I know that I can rely on. Some interactions have been positive and some negative. ... Don't drop a project just because one person in the project isn't positive." It seems likely that community interactions are key to successful student participation in HFOSS and instructor observations provide evidence of this.

Interviewees also talked about how their participation helped them learn about the real-world aspect of open source culture. One participant stated: "I didn't have any concept of what it meant for anyone around the world to be involved in co-creating something at the same time ... if they could get that understanding to students, even if they don't create open source, that would be a good thing ... raising awareness about what open source is and open principles."

4.1.2 Professional Practice as a Context for Computing Education. A second theme that emerged is that HFOSS education positions professional practice as a context for computing education. Some instructors don't have industry experience and therefore lack experience with collaborative, large scale projects. Involvement in HFOSS allows instructors to participate in current projects, using cutting-edge technologies, and model for their students how they approach new problems as they learn alongside their students.

There were 31 separate observations about professional practice and technical topics made by $12(63 \%)$ of the interviewees (four women, eight men). Four had no industry experience, four had 1-4 years of industry experience, two had 5-10 years of experience and two had $>10$ years of experience. The fact that half of the interviewees mentioned learning a new technology provides evidence that HFOSS is a rich learning environment as even those with extensive industry experience commented on learning a new technology. Simply learning a new tool is unsurprising for computing faculty. The insight in this theme is that HFOSS Education puts technology in a context of professional practice, and instructors can use that context to enhance learning. Table 4 shows an example code and coded data for the Professional Practice as a Context for Computing Education theme. Additional codes included Security, Tool knowledge, Open source workflow and Information literacy within a project.

When instructors spoke about professional practice in HFOSS education, two main sub-themes emerged: process and tools/ technologies. Instructors spoke about how incorporating H/FOSS into their courses gave them a better understanding of the work flow used in software engineering/development. One instructor noted

\begin{tabular}{|l|l|}
\hline Code & Raw Data \\
\hline $\begin{array}{l}\text { Software devel- } \\
\text { opment process }\end{array}$ & $\begin{array}{l}\text { "I wasn't aware of how extensive and dis- } \\
\text { tributed the software development base is and } \\
\text { how sophisticated the tools used are to coor- } \\
\text { dinate the software development process" }\end{array}$ \\
\hline
\end{tabular}

Table 4: Example Coding for Professional Practice as a Context

"I've gone from being kind of tersely familiar with open source software as a development area to being more involved in different ways of how people do software engineering, so now I feel better qualified to tell my students about best practices in this area." Another commented "I learned a lot about work flow, particularly in open source project[s], because I've never done professional software development." As academics, this was enlightening for several participants as they had no industry experience. By working in an HFOSS project they could see how the concepts they have taught play out in an open source project thus allowing them to provide real-world learning to students.

Several instructors commented that learning about software tools increased their ability to include those tools in their classroom. One instructor commented: "I've learned more tools and ways to incorporate them into my classes." and another stated when asked about what they learned "...hearing how others used tools (slack). How they use it." Comments like these reinforce the perception that HFOSS education provided a professional practice context that enabled instructors to enrich their teaching.

4.1.3 Instructors as Co-learners and Mentors. Perhaps the most interesting theme involved instructor reflections on themselves as an instructor, their transformation as an instructor, and their observations on the change of the instructor's role as a result of supporting student participation in HFOSS. Nine instructors (47\%) made 20 self-observations related to their growth as an instructor. Table 5 shows an example code and coded data for this theme.

\begin{tabular}{|l|l|}
\hline Code & Raw Data \\
\hline $\begin{array}{l}\text { More able } \\
\text { to release } \\
\text { control }\end{array}$ & $\begin{array}{l}\text { "... you're letting go of setting the end point for } \\
\text { students. I'm less sure of exactly where we're } \\
\text { going and where we'll end up." }\end{array}$ \\
\hline
\end{tabular}

Table 5: Example Coding for Instructor as Co-Learner The codes used for instructors as co-learners and mentors span topics that might be expected when employing a new instructional approach such as Instructor Learning Curve and Increase in Confidence. However, some interesting observations occurred under codes such as More Comfortable with Things not Going as Planned, More able to Release Control, Able to Admit not Knowing Something" and Better at Taking Risks. Additional tags included More Open to new Technologies, More Enthusiastic having Students Work in the Open, Real-World Impact, and More Willing to Expose Students to Real-World Complexity.

In analyzing these codes, five sub-themes emerged including confidence, ability to handle risk in the classroom, real-world applicability, understanding of teaching and/or learning, and motivation.

Four instructors commented on an increase in confidence or being more able to release control in the classroom. Some instructors also noted that they were more comfortable admitting to not knowing answers to student questions. One instructor commented that 
they were "... more willing to cede authority and admit that I don't know something." This provides some evidence that instructors are shifting from the "sage on the stage" to the "guide on the side" due to supporting student involvement in HFOSS.

Four instructors also remarked on an increased ability to handle risk in the classroom. One instructor commented that they were "...better at trying something, taking a risk,..." and another commented "I've gotten more comfortable with things not going as planned..." Another instructor indicated that they were more open to trying new technologies. These attitudes are often contrary to how instructors are used to teaching as many instructors have a fixed plan for a semester. The possibility of change is also contrary to the expectations that students have of their courses. However, this more fluid educational experience parallels the experiences students will have once they enter the workforce and therefore is valuable.

A third sub-theme that emerged was the real-world impact which included exposing students to code used in existing projects (not just academic examples), providing instructors with more than just the academic theory of software development, and working on a team to further develop a product already in use. One instructor indicated that it has given her/him that real-world context, not just the academic side. Another commented that s/he had "... gotten much more explicit with students that my learning goal for them is to learn how to work through mucky problems."

Four instructors provided evidence of greater understanding of teaching and/or learning with one instructor commenting on the learning curve by stating "It is such a learning process as a professor, it took me a while to get to the point to getting students to contribute." Another indicated that $\mathrm{s} /$ he felt "...we're at the very early stage of learning how software development should be taught - working on a team to develop product we can trust ..." One instructor commented on student capacity to learn stating "What I learned is how far students could go on their own if you mentor them well; when they graduated, they could write code better than the profs by far."

Perhaps the most interesting instructor comments were related to instructor motivation. Three instructors commented on how student participation in HFOSS transformed their teaching and was inspiring, with one instructor with more than 10 years experience as a computing educator stating that their teaching was "transformed". Another instructor, also with more than 10 years experience as a computing educator, commented about how proud s/he was at their own learning stating "... I learned a lot and was proud that I learned about it." It is also interesting to note that there were no negative comments related to instructor motivation.

\subsection{Resources for HFOSS Education}

This section presents emergent themes that help to answer research question 2: What resources did instructors believe were most important to their effort in HFOSS education? As discussed earlier, there is considerable evidence of the value of HFOSS education for students. In addition, the prior section indicates that there are interesting positive impacts of HFOSS education for the instructors. At the same time, it has been clear for some time that there is a significant learning curve for instructors as well as students pursuing HFOSS participation. The interviews provided several insights related to the resources instructors found helpful in navigating that learning curve and getting started in HFOSS education.

4.2.1 Working with Other Faculty. - The learning curve for HFOSS education is partly technical, with faculty needing to learn HFOSS culture, tools, and processes. At the same time, many of the challenges in HFOSS education relate to pedagogy and curriculum issues. Given the changes in teaching method and perspective discussed earlier, it seems reasonable that instructors would turn to other instructors as they get started in HFOSS education. Table 6 shows an example code and coded data for this theme.

\begin{tabular}{|l|l|}
\hline Code & Raw Data \\
\hline $\begin{array}{l}\text { Faculty } \\
\text { at own } \\
\text { institution }\end{array}$ & $\begin{array}{l}\text { "... I did have another POSSE member here on } \\
\text { campo I felt lucky for that; we did talk about } \\
\text { what we could do..." }\end{array}$ \\
\hline
\end{tabular}

Table 6: Example Coding for Working with Other Faculty

Collaborations among faculty members involved both experienced and new HFOSS educators, and also groups of new educators working together to problem solve and to co-develop HFOSS learning activities. While these collaborations were described as useful, they did not seem to be sustained. As one instructor noted, "once we got busy during the fall semester, active collaboration stopped with that group of four or five."

4.2.2 Community Gatherings. - Even though long-term collaboration on a regular basis seems difficult to maintain, many of the faculty interviewed have found periodic re-connection with the HFOSS education community to be of value. The faculty interviewed had all attended an initial POSSE workshop and so had some contacts within the community. Many of them have supplemented this initial experience by attending follow-up workshops often held in conjunction with other conferences related to computing education. These gatherings have created a community of HFOSS educators, one of the success factors for adopting new teaching approaches reported by Simon [28]. Table 7 shows an example code and coded data for this theme.

\begin{tabular}{|l|l|}
\hline Code & Raw Data \\
\hline $\begin{array}{l}\text { Materials } \\
\text { sprints }\end{array}$ & ".... hard but useful work." \\
\hline
\end{tabular}

Table 7: Example Coding for Community Gatherings

Faculty also valued the ability to reach out to the community on an ad-hoc basis. One instructor noted the value of a "community of experts that are easy to contact which is already in place." Several of the instructors seemed to find this faculty community important to their success with HFOSS education. As one noted: "If I was on my own, I probably would have given up."

4.2.3 Shared Learning Materials. - Instructors always seem to struggle to find enough time to develop learning materials. The HFOSS education community has put considerable effort into applying open source principles to ease this struggle and promote sharing of learning materials. Materials are located at http:foss2serve.org and http:teachingopensource.org. Table 8 shows an example code and coded data for this theme. 


\begin{tabular}{|l|l|}
\hline Code & Raw Data \\
\hline $\begin{array}{l}\text { POSSE ma- } \\
\text { terials }\end{array}$ & "Learning activities were helpful." \\
\hline
\end{tabular}

Table 8: Example Coding for Shared Learning Materials

One instructor commented on teaching an HFOSS course that the shared learning materials provided "a lot of material I used in this course." Another instructor thought that the learning materials "were helpful" although s/he didn't use them directly but rather reported that they "influenced and inspired" creation of custom versions.

\section{DISCUSSION}

The results presented in the previous section demonstrate that incorporating HFOSS into the classroom is not just about what the students learn, it also has great impact on the instructors. Three themes related to the impact on instructors' approaches and perspectives that emerge from the qualitative analysis show striking aspects of that impact on instructors. HFOSS education motivates instructors to become HFOSS community participants and provides a richer context for framing computing education, particularly with regard to software development. Perhaps most interestingly, instructors find HFOSS education changing their role to co-learning and mentoring rather than more traditional lecturing. Instructors report that HFOSS education has helped them learn to be more flexible in the classroom and more comfortable with non-traditional classroom management. This release of control is necessary to provide students with real-world experiences that are frequently complex, unbounded, and have no easily identified answers. As a result of HFOSS use in classes, the instructors feel that they are better able to handle risk and unexpected developments while teaching. This evolution of instructor role in HFOSS education has similarity to results reported for other active learning approaches.

The interviews for this study were conducted with instructors who were one to several years into their HFOSS education efforts. Many of the instructors had little or no prior open source experience before incorporating it into their course. As discussed above, the comments from these faculty centered on instructors as HFOSS community participants. It is interesting to note that the research team has also observed cases where instructors guide learning for students without direct participation by the instructor in the HFOSS project. One area for future investigation is that these differing levels of instructor participation represent two points on the instructor learning curve. Initially, instructor participation is more important while the instructor is developing expertise in HFOSS. Later on, with more HFOSS expertise, the instructor's level of HFOSS community participation may be more variable while still allowing instructors to guide and support student learning within the complexities of an HFOSS project. This may imply that, with development of expertise, instructors can become more efficient at HFOSS education, which would help with sustainability of the approach.

In addition to instructor learning, it was interesting to note that instructors found the experience to be motivating and in at least some cases, transformed their teaching. This provides an encouraging indicator for widespread adoption of HFOSS education. Given that instructors have considerable control over teaching methods used in their classroom, this consideration of instructor motivation is important for any approach that changes the instructor role.
In considering the results based on the resources used by instructors to support HFOSS education and the manner in which they employed them, it is interesting to note that the mind set of open source appears to be influencing how instructors teach. One instructor mentioned "a big thing is now that when I'm thinking about a course, I ask is there some way that open source would be a good contribution to the course". In addition, instructors appear to be reflecting on what they see in HFOSS communities and integrating some of that culture into their teaching. For instance, remarks by faculty members appear to indicate that they are more inclined to develop a course within a community of HFOSS educators, rather than a more typical approach where an instructor develops and delivers a course on his or her own. As one instructor indicated "Also, when working with other people in POSSE, a lot of them have a lot more teaching experience than I have, so to see how they handle activities has opened up new possibilities for me..."

These positive experiences for instructors come with a learning curve. As the themes related to the second research question show, connection with other faculty, access to learning materials, and periodic HFOSS education community events help with this learning curve. Even so, the required effort remains an inhibitor to adoption, and an area of future effort for the HFOSS education community.

\subsection{Limitations}

By definition, the instructors in this study had participated in the Professors' Open Source Software Experience (POSSE) [9] workshop. The results of this study are descriptive of that population, and may not be representative of the wider academic community, but the study does demonstrate the potential benefits gained not just by the students but also the instructors.

The process of identifying themes that emerge from the volume of data generated by 24 instructor interviews is inherently subjective. To mitigate this issue, best practices ensuring the rigor of qualitative research [16] were followed including using pairs of researchers to code with frequent meetings to resolve differences.

\section{CONCLUSION AND FUTURE WORKS}

The emphasis of much of the work in HFOSS education, and related pedagogical approaches, is what the students learn. This study provides some interesting and encouraging insights from investigating the impact on instructors of the process of supporting their students' involvement in an HFOSS project. Despite the learning curve, instructors who have been successful are enthusiastic about the opportunities HFOSS education provides for them. Future areas of study include an analysis of instructor expectations and student successes, and further examination of ways to reduce the learning curve for instructors interested in HFOSS education.

\section{ACKNOWLEDGMENTS}

This material is based on work supported by the National Science Foundation under Grant Nos. DUE-1525039, DUE-1524877, and DUE-1524898. Any opinions, findings and conclusions or recommendations expressed in this material are those of the author(s) and do not necessarily reflect the views of the National Science Foundation (NSF). 


\section{REFERENCES}

[1] Judith Bishop, Carlos Jensen, Walt Scacchi, and Arfon Smith. 2016. How to Use Open Source Software in Education. In Proceedings of the 47th ACM Technical Symposium on Computing Science Education (SIGCSE '16). ACM, New York, NY, USA, 321-322. DOI : http://dx.doi.org/10.1145/2839509.2844665

[2] Grant Braught, John Maccormick, James Bowring, Quinn Burke, Barbara Cutler, David Goldschmidt, Mukkai Krishnamoorthy, Wesley Turner, Steven HussLederman, Bonnie Mackellar, and Allen Tucker. 2018. A Multi-Institutional Perspective on $\mathrm{H} / \mathrm{FOSS}$ Projects in the Computing Curriculum. ACM Trans. Comput Educ. 18, 2, Article 7 (July 2018), 31 pages. DOI : http://dx.doi.org/10.1145/3145476

[3] Moara Sousa Brito, Fernanda Gomes Silva, Christina von Flach G. Chavez, Debora C. Nascimento, and Roberto A Bittencourt. 2018. FLOSS in Software Engineering Education An Update of a Systematic Mapping Study. SBES '2018, Brazilian Symposium on Software Engineering - Education Track (2018).

[4] Darci Burdge, Gregory W. Hislop, and Joanna Klukowska. 2018. Open Source Student Clubs: (Abstract Only). In Proceedings of the 49th ACM Technical Symposium on Computer Science Education (SIGCSE '18). ACM, New York, NY, USA, 1070-1070. DOI : http://dx.doi.org/10.1145/3159450.3162186

[5] Heidi J.C. Ellis, Gregory W. Hislop, Monisha S. Pulimood, Becka Morgan, and Ben Coleman. 2015. Software Engineering Learning in HFOSS: A Multi-Institutional Study. 122nd Association for Engineering Education Annual Conference and Exposition (2015).

[6] Heidi J.C. Ellis, Gregory W. Hislop, Josephine Rodriguez, and Ralph A. Morelli. 2012. Student Software Engineering Learning via Participation in Humanitarian FOSS Projects. 119th Annual ASEE Conference and Exhibition (2012).

[7] Heidi J. C. Ellis, Gregory W. Hislop, Stoney Jackson, and Lori Postner. 2015 Team Project Experiences in Humanitarian Free and Open Source Software (HFOSS). Trans. Comput. Educ. 15, 4, Article 18 (Dec. 2015), 23 pages. DOI: http://dx.doi.org/10.1145/2684812

[8] Carnegie Foundation. 2010. The Carnegie Classification of Institutions of Higher Education (n.d.). About Carnegie Classification. (2010). http:// carnegieclassifications.iu.edu/2010/

[9] Red Hat. 2017. Open Source in Education. (2017). https://www.redhat.com/en/ about/open-source-education/educators

[10] Antti Herala, Erno Vanhala, Antti Knutas, and Jouni Ikonen. 2015. Teaching Programming with Flipped Classroom Method: A Study from Two Programming Courses. In Proceedings of the 15th Koli Calling Conference on Computing Education Research (Koli Calling '15). ACM, New York, NY, USA, 165-166. DOI : http://dx. doi.org $/ 10.1145 / 2828959.2828983$

[11] Gregory W. Hislop, Heidi J.C. Ellis, S. Monisha Pulimood, Becka Morgan, Suzanne Mello-Stark, Ben Coleman, and Cam Macdonell. 2015. A Multi-Institutional Study of Learning via Student Involvement in Humanitarian Free and Open Source Software Projects. In Proceedings of the Eleventh Annual International Conference on International Computing Education Research (ICER '15). ACM, New York, NY, USA, 199-206. DOI: http://dx.doi.org/10.1145/2787622.2787726

[12] Yanli Hu, Liang Bai, and Bin Ge. 2018. Online Behavior Analysis-based Blended Learning Model. In Proceedings of ACM Turing Celebration Conference - China (TURC '18). ACM, New York, NY, USA, 136-137. DOI : http://dx.doi.org/10.1145/ 3210713.3210748

[13] Zhewei Hu, Yang Song, and Edward F. Gehringer. 2018. Open-source Software in Class: Students' Common Mistakes. In Proceedings of the 40th International Conference on Software Engineering: Software Engineering Education and Training (ICSE-SEET '18). ACM, New York, NY, USA, 40-48. DOI : http://dx.doi.org/10.1145/ 3183377.3183394

[14] Clayton Lewis. 2007. Attitudes and Beliefs About Computer Science Among Students and Faculty. SIGCSE Bull. 39, 2 (June 2007), 37-41. DOI : http://dx.doi. org/10.1145/1272848.1272880

[15] Hanna Mäenpää, Sasu Tarkoma, Samu Varjonen, and Arto Vihavainen. 2015 Blending Problem- and Project-Based Learning in Internet of Things Education Case Greenhouse Maintenance. In Proceedings of the 46th ACM Technical Symposium on Computer Science Education (SIGCSE '15). ACM, New York, NY, USA, 398-403. DOI : http://dx.doi.org/10.1145/2676723.2677262
[16] Nicholas Mays and Catherine Pope. 1995. Qualitative Research: Rigour and qualitative research. BMF 311, 6997 (1995), 109-112. DOI : http://dx.doi.org/10. 1136/bmj.311.6997.109 arXiv:https://www.bmj.com/content

[17] M.; Gius E.; Jacke J. Meister, J.C.; Petris. 2016. CATMA 5.0 [software for text annotation and analysis]. (2016). http://www.catma.de

[18] Becka Morgan and Carlos Jensen. 2014. Lessons Learned from Teaching Open Source Software Development. In Open Source Software: Mobile Open Source Technologies, Luis Corral, Alberto Sillitti, Giancarlo Succi, Jelena Vlasenko, and Anthony I. Wasserman (Eds.). Springer Berlin Heidelberg, Berlin, Heidelberg, 133-142.

[19] Debora M. C. Nascimento, Roberto Almeida Bittencourt, and Christina Chavez. 2015. Open source projects in software engineering education: a mapping study. Computer Science Education 25, 1 (2015), 67-114. DOI : http://dx.doi.org/10.1080/ 08993408.2015.1033159 arXiv:https://doi.org/10.1080/08993408.2015.1033159

[20] Lijun Ni, Tom McKlin, and Mark Guzdial. 2010. How Do Computing Faculty Adopt Curriculum Innovations?: The Story from Instructors. In Proceedings of the 41st ACM Technical Symposium on Computer Science Education (SIGCSE '10). ACM, New York, NY, USA, 544-548. DOI : http://dx.doi.org/10.1145/1734263.1734444

[21] Jacob Perrenet. 2009. Differences in Beliefs and Attitudes About Computer Science Among Students and Faculty of the Bachelor Program. In Proceedings of the 14th Annual ACM SIGCSE Conference on Innovation and Technology in Computer Science Education (ITiCSE '09). ACM, New York, NY, USA, 129-133. DOI : http://dx.doi.org/10.1145/1562877.1562920

[22] G. H. L. Pinto, F. F. Filho, I. Steinmacher, and M. A. Gerosa. 2017. Training Software Engineers Using Open-Source Software: The Professors' Perspective. In 2017 IEEE 30th Conference on Software Engineering Education and Training (CSEE T). 117-121. DOI : http://dx.doi.org/10.1109/CSEET.2017.27

[23] Leo Porter, Dennis Bouvier, Quintin Cutts, Scott Grissom, Cynthia Lee, Robert McCartney, Daniel Zingaro, and Beth Simon. 2016. A Multi-institutional Study of Peer Instruction in Introductory Computing. In Proceedings of the 47th ACM Technical Symposium on Computing Science Education (SIGCSE '16). ACM, New York, NY, USA, 358-363. DOI : http://dx.doi.org/10.1145/2839509.2844642

[24] Leo Porter, Cynthia Lee, Beth Simon, and Mark Guzdial. 2017. Preparing Tomorrow's Faculty to Address Challenges in Teaching Computer Science. Commun. ACM 60, 5 (April 2017), 25-27. DOI : http://dx.doi.org/10.1145/3068791

[25] Lori Postner, Heidi J.C. Ellis, and Gregory W. Hislop. 2018. A Survey of Instructors' Experiences Supporting StudentLearning Using HFOSS Projects. In Proceedings of the 49th ACM Technical Symposium on Computer Science Education (SIGCSE '18). ACM, New York, NY, USA, 203-208. DOI : http://dx.doi.org/10.1145/3159450. 3159524

[26] Alex D. Radermacher and Gursimran S. Walia. 2011. Investigating the Effective Implementation of Pair Programming: An Empirical Investigation. In Proceedings of the 42Nd ACM Technical Symposium on Computer Science Education (SIGCSE '11). ACM, New York, NY, USA, 655-660. DOI : http://dx.doi.org/10.1145/1953163. 1953346

[27] Samuel A. Rebelsky. 2015. HFOSS-Lite in CS2: Incorporating the Ushahidi Crowdmapping Program in a Data Structures and Algorithms Course (Abstract Only). In Proceedings of the 46th ACM Technical Symposium on Computer Science Education (SIGCSE '15). ACM, New York, NY, USA, 514-514. DOI : http://dx.doi.org/10.1145/2676723.2691885

[28] Beth Simon, Elizabeth Bales, William G. Griswold, and Stephen Cooper. 2011. Case Study: Faculty Professional Development Workshops for Innovation Diffusion. In Proceedings of the 42Nd ACM Technical Symposium on Computer Science Education (SIGCSE '11). ACM, New York, NY, USA, 673-678. DOI : http://dx.doi.org/10.1145/ 1953163.1953350

[29] Steven J Taylor, Robert Bogdan, and Marjorie DeVault. 2015. Introduction to Qualitative Research Methods: A Guidebook and Resource. John Wiley \& Sons.

[30] Saijing Zheng, Pamela Wisniewski, Mary Beth Rosson, and John M. Carroll. 2016. Ask the Instructors: Motivations and Challenges of Teaching Massive Open Online Courses. In Proceedings of the 19th ACM Conference on ComputerSupported Cooperative Work \& Social Computing (CSCW'16). ACM, New York, NY, USA, 206-221. DOI : http://dx.doi.org/10.1145/2818048.2820082

[31] Bizot B. zweben, S. 2017. 2017 CRA Taulbee Survey. (2017). https://cra.org/ wp-content/uploads/2018/05/2017-Taulbee-Survey-Report.pdf 